\title{
A DISSEMINAÇÃO DO SOFTWARE LIVRE GCOMPRIS EM OFICINAS PARA CRIANÇAS EM ATIVIDADE DE REABILITAÇÃO COGNITIVA
}

\author{
Gilmário Barbosa dos Santos ${ }^{1}$ \\ Janine Kniess ${ }^{2}$ \\ Juliane Cristine Koerber Reis ${ }^{3}$ \\ Eliane Korn ${ }^{4}$ \\ Cleide Aparecida Hoffmann Bernardes ${ }^{5}$
}

\begin{abstract}
RESUMO: Este texto relata a experiência de disseminação do Software Livre, realizada pelo grupo de extensão Colmeia do Departamento de Ciência da Computação da Universidade do Estado de Santa Catarina (UDESC). O conceito de Software Livre é brevemente discutido, bem como as atividades do grupo Colmeia, de onde são destacados os relatos das oficinas para divulgação do Software Livre educacional, denominado GCompris. Essas oficinas ocorreram durante o ano de 2012, nas dependências da Universidade do Estado de Santa Catarina, em Joinville, e tiveram como público alvo as crianças em reabilitação cognitiva, atendidas pela Associação de Assistência à Criança Deficiente (AACD) de Joinville-SC, bem como seus pais e cuidadores.
\end{abstract}

PALAVRAS-CHAVE: Software livre. Hardware livre. Organização social.

The dissemination of the free software GCompris in workshops for children in cognitive rehabilitation

\begin{abstract}
This paper reports the dissemination of a free software experienced by the group of extension research called Colméia, from the Science Computer Department of the University of the State of Santa Catarina (UDESC). The free software concept is briefly discussed, as well as the activities done by the group, from where the reports of workshops done for dissemination of the educational free software, GCompris, are taken. These workshops took place during 2012 at the University of the State of Santa Catarina, in Joinville, Brazil. They aimed to reach children in cognitive rehabilitation assisted by the Disabled Children Assistance Association (Associação de Assistência à Criança Deficiente - AACD) from the same city cited above, as well as their parents and caregivers.
\end{abstract}

KEYWORDS: Free software. Free hardware. Social organization.

\footnotetext{
${ }^{1}$ Doutor em Engenharia Elétrica com ênfase em Engenharia de Computação pela Universidade de Campinas, professor do Departamento de Ciência da Computação da Universidade do Estado de Santa Catarina (gilmario@joinville.udesc.br).

${ }^{2}$ Doutora em Ciência da Computação pela Universidade Federal Fluminense, professora adjunta do Departamento de Ciência da Computação da Universidade do Estado de Santa Catarina (janine@joinville.udesc.br)

${ }^{3}$ Mestre em Distúrbios do Desenvolvimento pela Universidade Presbiteriana Mackenzie, psicóloga na Associação de Reabilitação da Criança Deficiente (ARCD) (psicojckr@gmail.com).

${ }^{4}$ Especialista em Psicopedagogia Clínica e Institucional pela Faculdade Padre João Bagozzi, pedagoga da Associação de Assistência à Criança Deficiente (AACD) e professora alfabetizadora da rede municipal de ensino de Joinville-SC (eliane@arcd.org.br).

${ }_{5}^{5}$ Mestranda em Educação pela Universidade da Região de Joinville, pedagoga na Associação de Assistência à Criança Deficiente (AACD) (cleidepedago@gmail.com)
} 


\section{INTRODUÇÃO}

O conceito de liberdade, implícito no software livre (SL), está associado à abertura dos códigosfontes ${ }^{6}$ para a livre manipulação/alteração dos programas de computador (GNU Project, 2013). De maneira similar, o conceito de hardware livre (HL) tem como base a abertura dos projetos dos circuitos eletrônicos. Principalmente devido a essas características, a adoção do SL e/ou HL apresenta importantes implicações socioeconômicas. Especialmente o setor público se beneficia, reduzindo ou eliminando os custos de renovação/aquisição de licenças (usualmente cobradas) para uso dos softwares comerciais, implicando na diminuição do controle determinado pela indústria desse tipo de software, cujos programas não são "abertos". Um raciocínio aproximado pode ser aplicado ao campo do HL.

Diferentemente de outras iniciativas semelhantes de extensão universitária, o grupo Colmeia ${ }^{7}$ (Departamento de Ciência da Computação / Universidade do Estado de Santa Catarina - UDESC) não limita o seu campo de ação apenas a projetos de inclusão digital de contingentes desassistidos. Além de viabilizar o acesso à tecnologia de informática, o grupo se preocupa com a ampliação de horizontes, criando alternativas ao predominante uso e desenvolvimento do software comercial tradicional. Nesse sentido, a atuação do grupo é dividida em diversas ações (palestras, minicursos, participações em congressos, veiculação na mídia, organização de eventos, parcerias etc.), disseminando não apenas a "cultura de desenvolvimento livre", como também o próprio uso do SL e do HL.

Na seção que se segue estão detalhadas as principais ações de extensão do grupo Colmeia. Posteriormente, é feita uma breve descrição acerca do SL e motivação para sua adoção, além do relato da experiência de extensão universitária junto a uma organização social (a Associação de Assistência à Criança Deficiente - AACD) na cidade de Joinville-SC. As considerações finais são apresentadas na última seção.

\section{Motivação para a adoção do software livre}

Apesar da crescente aquisição de computadores pessoais, o custo do software ainda é relativamente alto para os padrões de renda nacional. Isso é demonstrado pelos altos índices de pirataria de software, conforme frequentemente noticiado na mídia. Nesse quesito, há um forte potencial para a adoção do SL, principalmente na forma de sistemas operacionais, navegadores de internet, processadores de vídeo, de texto e planilhas.

A adoção de soluções baseadas na cultura do SL implica na redução de custos de manutenção, desenvolvimento e aquisição de programas. O contato com essas tecnologias de código/projeto aberto amplia o horizonte de desenvolvedores e usuários, trazendo possibilidades não percebidas anteriormente. Já existem produtos bastante depurados e estáveis, contando com uma ampla comunidade de usuários e desenvolvedores, tanto no âmbito doméstico quanto em empresas. Há, ainda, casos de uso do SL para fins comerciais em diversos setores, em muitos casos, visando à

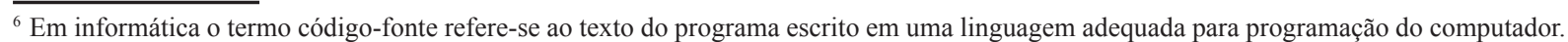
${ }^{7}$ Grupo de Pesquisa em Software e Hardware Livre da Universidade do Estado de Santa Catarina - UDESC em Joinville (www.colmeia.udesc.br). 
mera redução de custos de produção.

Em um nível um pouco maior de assimilação do paradigma de liberdade (há uma aura ideológica envolvendo o conceito de software livre), o próprio usuário pode participar do desenvolvimento ou aprimoramento do software utilizado. Isso é possível e existem diferentes níveis de participação, os quais exigem níveis variados de conhecimentos. Por exemplo, a simples troca de experiências nos fóruns de discussão na internet podem suscitar novas funcionalidades ao software, outra opção seria a tradução de manuais de uso, o que exigiria um nível de participação mais ativa.

Os produtos desenvolvidos com tecnologias de código/projeto aberto são mais acessíveis ao consumidor e o custo para atualização desses produtos, a longo prazo, é relativamente mais baixo. Trata-se de um aspecto importante não apenas para o setor público, mas também para algumas organizações sociais, as quais normalmente não dispõem de orçamento para custear a aquisição e atualização de softwares comerciais, no ritmo das suas demandas. Além disso, essas organizações, muitas vezes, não possuem corpo técnico voltado à informática e desconhecem as aplicações do SL. O contato com instituições pode ser bastante produtivo e surpreendente. É o que aconteceu no caso da interação com a AACD, especialmente durante as oficinas de SL, realizadas com pacientes de reabilitação desta instituição em Joinville-SC.

Por meio de uma equipe interdisciplinar de médicos e terapeutas especializados, a AACD atende a pacientes com deficiência física e busca promover a sua inclusão social. São atendidos pacientes com sequela de paralisia cerebral (PC) menores de 18 anos, mielomeningocele, amputados, pacientes com sequela de acidente vascular cerebral (AVC), traumatismo crânio encefálico (TCE), pacientes com lesão medular e outras patologias que trazem prejuízo ao sistema neuropsicomotor e que podem, efetivamente, apresentar resultados positivos com o programa de reabilitação oferecido pela AACD-SC.

\section{Uso do GCompris na reabilitação cognitiva de crianças}

Rosa Costa (2006) descreve "cognição" como uma complexa coleção de funções mentais, dentre as quais são citadas: monitoramento, julgamento, planejamento e solução de problemas. Considerando a importância dessas habilidades no cotidiano, quaisquer danos cerebrais que as afetem vêm a causar significativo prejuízo à qualidade de vida do paciente, sendo muitas vezes uma necessidade vital recuperar a capacidade cognitiva afetada.

Por sua vez, as estratégias de reabilitação cognitiva têm sido cada vez mais apoiadas em técnicas computacionais, ou seja, o uso dos programas de computador está cada vez mais presente nessa área de aplicação (exemplos podem ser encontrados nos trabalhos de Rosa Costa e colaboradores (2000) e Nascimento e colaboradores (2008)). Essa percepção, associada aos resultados obtidos nas oficinas de divulgação do SL, é que respalda o uso do GCompris como ferramenta na reabilitação cognitiva, conforme será evidenciado nos próximos parágrafos.

Durante a realização das atividades do grupo Colmeia, no decorrer do ano de 2011, foi realizado o 
recondicionamento de computadores desktop para doação. Parte desses computadores foi doadaàAACDSC com o sistema operacional Ubuntu instalado e, dentre outros, o software livre educativo GCompris (Figura 1). A intenção inicial do grupo Colmeia era prover a instituição com computadores a serem utilizados por seus pacientes e para fins administrativos.

Devido a questões de calendário, naquela ocasião não houve tempo para a preparação da equipe de monitoria para orientação dos pacientes e, por conta disso, esperava-se certa resistência ao contato com os softwares instalados. Entretanto, a aceitação dos usuários foi excelente, com destaque para o próprio GCompris. Assim, foi atestada a qualidade desse SL, um software de fácil compreensão e manipulação, atingindo um público de diferentes faixas etárias (crianças, adolescentes e adultos).

Figura 1 - Algumas telas de jogos oferecidos pelo software livre GCompris.

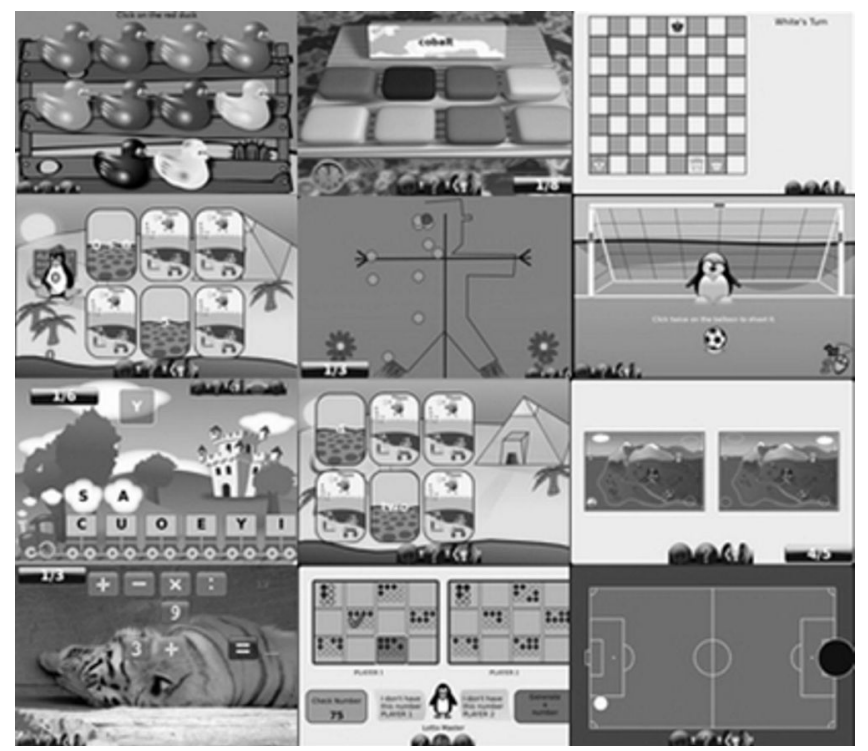

Fonte: Produção dos próprios autores.

Atualmente, esses computadores (contendo o software GCompris) estão dispostos na sala de espera da instituição. Os pacientes fazem uso deles equipamentos enquanto aguardam pela realização de suas terapias. Observa-se que o interesse por este software é frequente. Ou seja, o usuário tem um primeiro contato para conhecê-lo e ao longo das semanas continua utilizando-o, de maneira prazerosa.

Além do acesso livre na sala de espera, o GCompris é utilizado nos setores de Pedagogia e Psicologia da instituição como uma das ferramentas de estimulação e reabilitação cognitiva. Nestes setores, as atividades são escolhidas e planejadas pelo profissional conforme os objetivos propostos para cada paciente. São realizadas tarefas mediadas, com a finalidade de atingir as metas de estimulação/reabilitação cognitiva, bem como, desenvolver habilidades como percepção visual, organização espacial, raciocínio lógico, operações aritméticas, leitura, escrita, entre outros, com a intenção de otimizar a aprendizagem escolar e social.

Por ser um software livre, acredita-se que seu alcance pode vir a ser ainda maior. Por conta da abertura do código-fonte, há possibilidade de modificações no GCompris, de acordo com as 
necessidades dos usuários. A quantidade de estímulo visual/auditivo, bem como alterações nos níveis de dificuldades, por exemplo, podem tornar o GCompris mais funcional para os pacientes.

A aceitação dos usuários atestou a qualidade desse software, especialmente quanto à funcionalidade e usabilidade. Diante disso, foram implementadas oficinas, durante o ano de 2012, para a consolidação do uso do GCompris para um público com necessidades de reabilitação cognitiva (Figura 2 e Figura 3). Essas oficinas foram realizadas nas dependências do Departamento de Ciência da Computação da UDESC, em Joinville, e contaram com a presença de pacientes da AACD-Joinville-SC (crianças e adolescentes), seus acompanhantes/cuidadores, além de profissionais da AACD.

Figura 2 - Atendimento individual durante oficina de divulgação do SL GCompris para crianças pacientes da AACD em Joinville-SC (2012).

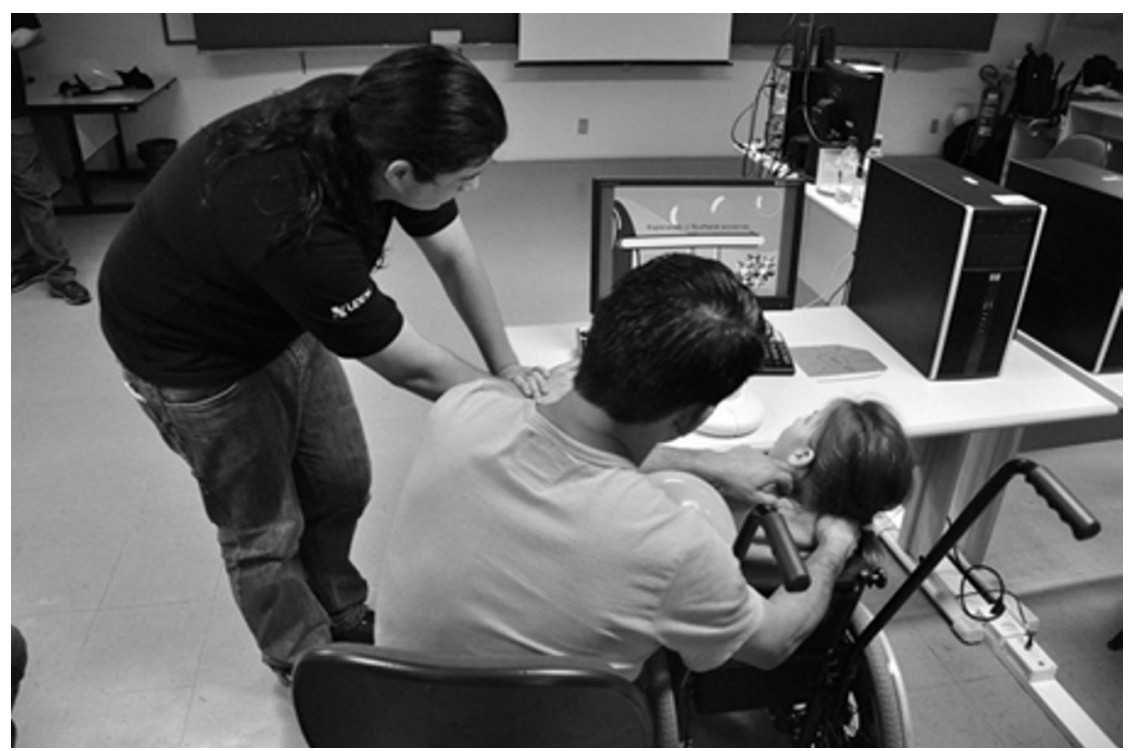

Fonte: Jonas Porto (Assessoria de Imprensa/UDESC).

Além da prazerosa socialização propiciada, os pacientes (e cuidadores) que compareceram às oficinas do Colmeia/AACD tiveram a oportunidade de conhecer os conceitos de SL e HL, bem como o sistema operacional Linux na distribuição Ubuntu. Em seguida, foi apresentado o SL GCompris e seus recursos. Esse software mescla atividades de programas de exercício-e-prática com a abordagem de jogos. O resultado lúdico desta combinação motiva o paciente, fazendo desse software uma importante ferramenta para a estimulação para fins de reabilitação cognitiva.

As experiências com o GCompris demonstraram que esse instrumento pode fazer parte de um programa global de estimulação, trazendo resultados positivos no processo de reabilitação cognitiva. Aliada à aprendizagem e ao desenvolvimento de estratégias compensatórias, o software GCompris oportuniza ao paciente a exploração e enriquecimento de suas potencialidades. Por conta disso, além de conhecer os recursos do software, ao final de cada oficina foram oferecidas cópias do GCompris em CDs, orientação quanto ao seu funcionamento e o suporte do grupo Colmeia - para o caso de futuras dúvidas quanto a instalação/uso do sistema. 
Figura 3 - Apresentação de palestra durante oficina de divulgação do SL GCompris para crianças pacientes da AACD em Joinville-SC (2012).

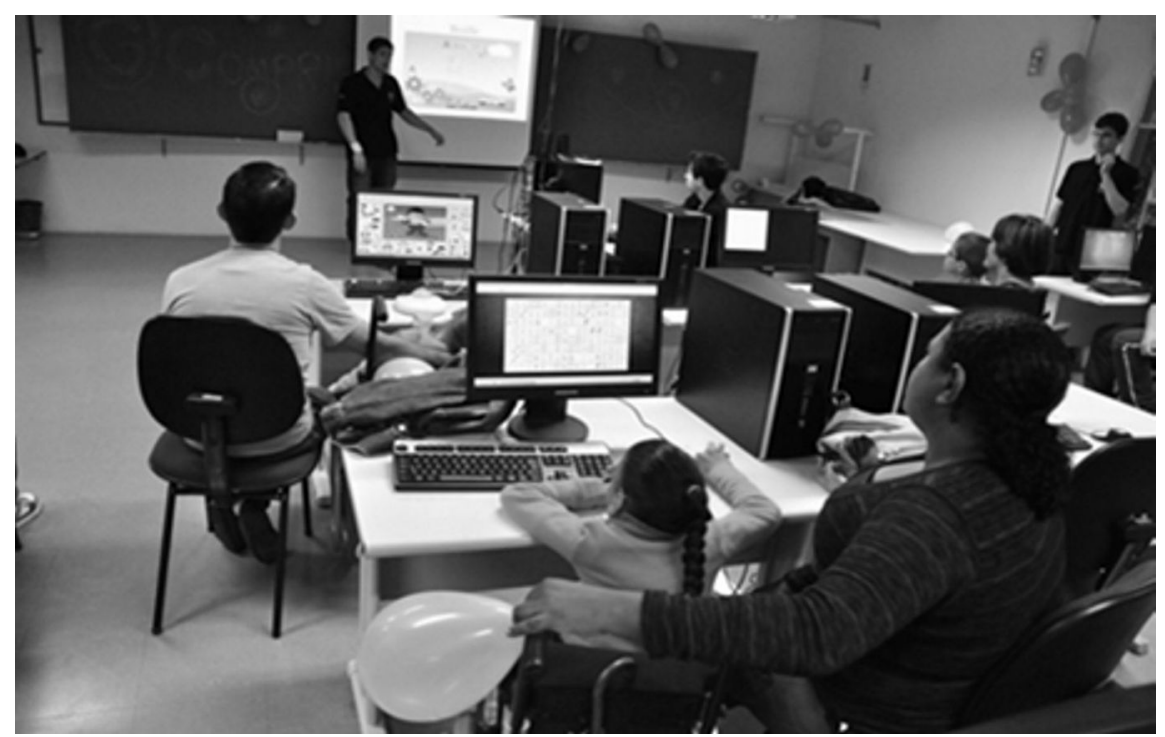

Fonte: Jonas Porto (Assessoria de Imprensa/UDESC).

A disponibilidade gratuita do GCompris, para uso doméstico pode trazer benefícios significativos, pois, com essa possibilidade, o processo de reabilitação não fica restrito ao ambiente da AACD.

A avaliação das oficinas foi realizada por meio da consulta aos participantes e por opiniões colhidas dos membros do corpo técnico da AACD, que participaram dos eventos. Os relatos foram positivos e, com isso, os membros do grupo Colmeia sentem-se motivados e julgam importante reeditar eventos como esses nos próximos anos, incluindo novas perspectivas para o uso do SL em geral, particularmente do GCompris.

\section{CONSIDERAÇÕES FINAIS}

A disseminação do software livre apresenta um forte potencial a ser explorado na prática da extensão universitária. O grupo de extensão Colmeia (Departamento de Ciência da Computação UDESC/Joinville-SC) identificou esse potencial e as pessoas e/ou instituições de fato têm se beneficiado por meio das ações realizadas.

As experiências com as oficinas promovidas pelo grupo Colmeia proporcionaram aos pacientes da AACD - SC e a seus cuidadores, não apenas a apresentação de uma ferramenta que auxilia no processo de estimulação e reabilitação cognitiva, como também exerceu uma ação de inclusão digital, apresentando-se como um universo de possibilidades, atrativo e funcional.

Nesse sentido, a gratuidade, aliada à qualidade do SL, configuraram-se como oportunidades de acesso e uso desta ferramenta, a usuários de diferentes faixas etárias, interesses e necessidades. Com a disponibilização dos CDs para uso doméstico dos jogos contidos no GCompris e com o suporte oferecido pelo grupo Colmeia, percebe-se um efeito positivo e imediato, decorrente do 
acesso gratuito a este programa educativo e terapêutico, pois amplia o tratamento dos casos que necessitam de estimulação cognitiva.

As ações do grupo Colmeia serão continuadas nos próximos anos, sempre com o interesse em fornecer mais informação acerca do SL/HLa todos os públicos, especialmente às instituições de caráter social.

Considerando a satisfação do público que frequentou as oficinas do GCompris, espera-se que sejam possíveis outras ações junto aos pacientes da AACD, não apenas no contexto do GCompris, mas também na divulgação de outras soluções em SL, funcionais, gratuitas, de qualidade e que auxiliem no processo de estimulação e reabilitação de seus pacientes.

\section{AGRADECIMENTOS}

De maneira especial, agradecemos a participação das crianças, pais e demais acompanhantes que prestigiaram as oficinas. Agradecemos, também, às instituições envolvidas, à AACD $\mathrm{SC}$, à UDESC/DCC, bem como aos estudantes (bolsistas e voluntários) do grupo Colmeia.

\section{REFERÊNCIAS}

GNU Project - GNU Operating System. O que é Software Livre. Disponível em: < www.gnu. org/philosophy/free-sw.html>. Acesso em: 14 jun. 2013.

NASCIMENTO, D. B.; CARVALHO, G. F. J.; COSTA, R. M. E. M. ReabRA: reabilitação cognitiva através de uma aplicação de realidade aumentada. In: WORKSHOP DE REALIDADE VIRTUAL E AUMENTADA - WRVA, 5., 2008, Bauru. Anais..., Bauru: Universidade Estadual Paulista, 2008. Disponível em: <www2.fc.unesp.br/wrva/artigos/50466.pdf>. Acesso em: 14 jun. 2013.

COSTA, R. M. E. M.; CARVALHO, L. A. V.; ARAGON, D. F. Novas tecnologias computacionais na reabilitação cognitiva. In: SIMPÓSIO ARGENTINO DE INFORMÁTICA Y SALUD SADIO, 3.,Buenos Aires, 2000, Anais..., Buenos Aires, 2000. Disponível em: < www.unifra.br/ professores/anabonini/tecnologias_reabilitacao.pdf>. Acesso em: 14 jun. 2013.

COSTA, R. M. E. M. A realidade virtual nas neurociências. In: TORI, R.; KIRNER,C.; SISCOUTO, R. (Org.). Fundamentos e tecnologia de realidade virtual e aumentada. Porto Alegre: Sociedade Brasileira de Computação, 2006. Disponível em: <http://www.pcs.usp. br/ interlab/Fundamentos_e_Tecnologia_de_Realidade_Virtual_e_Aumentada-v22-11-06.pdf $>$. Acesso em: 14 jun. 2013.

SANTOS, F. H. Reabilitação neuropsicológica pediátrica. Psicol. Ciênci. Prof., Brasília, DF, v. 25, n. 3, p. 450-461, set. 2005. Disponível em $<$ http://pepsic.bvsalud.org/scielo.php?pid=S141498932005000300009\&script=sci_arttext>. Acesso em: 14 jun. 2013.

Submetido em 20 de fevereiro de 2013. Aprovado em 13 de junho de 2013. 